\title{
THE USE OF DEM SIMULATION FOR CONFIRMING THE PROCESS OF PARTICULATE MATERIAL MIXING
}

\author{
Peter Peciar*, Oliver Macho, Roman Fekete, Marián Peciar
}

\author{
Slovak University of Technology in Bratislava, Faculty of Mechanical Engineering, Institute of Process \\ Engineering, Námestie Slobody 17, 812 31, Bratislava, Slovakia \\ * corresponding author: peter.peciar@stuba.sk
}

\begin{abstract}
At the present time in the research of particulate materials, computer methods that work independently with individual particles are coming to the forefront. One such method is the Discrete Element Method - DEM, which is already widely used. Its usage, however, is complex, mostly due to the input data - what the properties of the researched material are, plus their interaction in processes. And despite the progress, it is still always necessary to validate the experimental equipment and to verify the individual simulations by an experimental measuring or by theoretical knowledge. This study focuses on the verification of the simulation of the mixing of a particulate material with the help of the DEM method, whereby simulations are compared with an experimental measurement and theoretic calculations. The theoretical calculation was carried out by the Novosad model, while the experimental material was granulated polyethylene with strictly defined mechanical-physical properties.
\end{abstract}

KEYwords: particulate material, mixing, theoretical knowledge, experimental measurements, DEM method.

\section{INTRODUCTION}

Mixing of a particulate material is a very old process, but nonetheless relatively little studied. Research in this area is progressing quite slowly. Studies dealing with the effects of the forces acting during the process of mixing appear only sporadically and the majority of them focuses on the kinetics of mixing and computer simulations without any verification by experiments [1, 2.

Among the pioneers in the field of mixing belong D. Bagster and J. Bridgwater 3 5, who undertook measuring of the forces acting on a flat mixing element. In their first experiments, they used a static blade and the chamber performed a straight-line motion. They later designed a new mixing apparatus with a static glassed chamber in the shape of a prism. The blade moved in a reciprocating motion in a horizontal direction in the material while the station, equipped with measuring elements, recorded forces in both the horizontal and the vertical directions.

The results of these researches pointed to a dependence of the measured forces on the properties of the particulate material, specifically the bulk density, material particle density, internal friction angle, wall friction angle, material porosity and blade geometry. This dependence was formulated into the relation

$$
\frac{H}{\rho(1-\epsilon) g L B^{2}}=f\left(\frac{z}{B}, \frac{L}{B}, \frac{v^{2}}{g B}, \varphi_{i}, \varphi, \epsilon\right),
$$

where $H$ is the horizontal force acting on the blade, $\rho$ the particle density of the material, $\epsilon$ the porosity of the material, $g$ the gravitational acceleration, $L$ the blade length, $B$ the blade height, $z$ the immersion of the blade in the mixed material, $v$ the blade speed, $\varphi_{i}$ the internal friction angle, $\varphi$ the wall friction angle between particulate material and blade material.

Their experiments showed that the size of the horizontal force is not dependent on the porosity of the material or blade speed. The authors also found out that the value of the angle of the force from the horizontal, by which the blade acts on the mixed material, is approximately the same as the value of the wall friction angle of the particulate material and the blade material.

In his work, J. Novosad [6, 7] carried out an in experimental monitoring of a course of mixing of several kinds of bulk solids with a blade mixer. In his experiments, he used glass cylindrical chambers in a vertical arrangement. His contribution was mainly the usage of mechanical properties of mixed particulate materials in relation to the calculation of mutually strong effects of mixed particulate materials and the mixing element. He found out that the most important variable characterizing particulate materials was the role of the internal and wall friction and bulk density.

K. Kravárik 8 8 worked on the explanation of the force effects of particulate materials on a flat blade going through the layer of its batch and also on methods of the calculation of the forces acting on a flat blade of rectangular shape moving at a constant speed in a horizontal direction.

The investigated materials included granulated fertilizer, granulated polyethylene and sand. As the parameters according to which he monitored the dependence of the amount of force acting on the blade, he used the internal friction angle, the wall friction 
angle of the blade material, the bulk density, the height and length of the blade, the depth of the blade immersion in the material and the blade speed.

For a theoretical clarification of the formation of the shear zone in a particulate material before the blade, he compared various calculation models and, in experimental conditions, studied the shape of the shear zone before the advancing blade from the Novosad model and formulated a dimensionless, critical dependence to calculate the force acting on the blade:

$$
\frac{H}{\rho_{S} g L B^{2}}=k_{1}\left(\frac{z}{B}\right)^{k_{2}}\left(\frac{v^{2}}{g B}\right)^{k_{3}}\left(\frac{\tan \varphi_{i}}{\tan \varphi}\right)^{k_{4}},
$$

where $H$ is the horizontal force acting on the blade, $\rho_{S}$ the bulk density of the particulate material, $g$ the gravitational acceleration, $L$ the blade length, $B$ the blade height, $z$ the immersion of the blade in the mixed material, $v$ the blade speed, $\varphi_{i}$ the internal friction angle, $\varphi$ the wall friction angle between the particulate material and blade material, $k_{1}, k_{2}, k_{3}$, $k_{4}$ the coefficients of critical dependencies for the calculation of the dimensionless force.

On the basis of the results achieved, the author concluded that the dimensionless force significantly depends on the dimensionless immersion of the blade. The effect of the material's internal friction angle is relatively significant and the effect of the dimensionless speed of the blade in the given extent has a serious effect on the size of the dimensionless horizontal force. The influence of the wall friction angle was eliminated by creating a static wedge area in front of the blade.

At the present time, a simulation of tasks in a variety of programs with the use of several computational methods is gaining prominence. However, for investigating particulate materials and mixing elements, the DEM method remains the most used throughout the world. In 1997, Y. Muguruma, T. Tanaka, S. Kawatake and Y. Tsuji [9] experimentally studied the three-dimensional motion of particles in a rotation mixer with two baffles at different degrees of filling of the mixing chamber. They then simulated the experiments with the DEM method. They found out that the mixing curve, which coordinates the level of premixing and time through the DEM method, was in an accordance with the foregoing experiments. The result was the emergence of two distinct stages on the mixing curve.

M. S. Siraj, S. Radl, B. J. Glasser and J. G. Khinast in their work [10, point out the influence of turning the blade travelling through the particulate material by the DEM method. The mixing equipment was made up of a prism-shaped static chamber with a moving blade with a variable slope. As the modelling material, glass balls of different diameters were used. The authors performed the simulation for several angles of turning the blade and for different particle size ratios. They expected that, to a certain degree, some particles in the bulk layers would show a tendency to rise to the top and others fall to the bottom, which represents segregation. If the larger particles rose to the top layer, they tended to remain there at the beginning of the experiment. With further simulations, they registered a significant influence from the material friction on the chamber walls. A large difference in the mixing of the particulate material was mainly visible with smaller angles of the blade slope $\left(60^{\circ}\right.$, $90^{\circ}$ ), while at an angle $140^{\circ}$, there was no significant change between the simulation that included wall friction and that which did not. In their simulations, they also tried to identify the normal and the tangential forces acting between the particles. The experiment showed that the extent of the normal and tangential forces was indirectly proportional to the slope angle of the blade, such that the higher forces were for the slope angles of the blade less than $90^{\circ}$ and the lower forces were for the slope angles above $90^{\circ}$.

In their work, G. R. Chandratilleke, A. B. Yu and J. Bridgwater attempted [11] to verify, with the use of the DEM method, the studies of D. F. Bagster and J. Bridgwater. On the basis of the dimensional analysis of the horizontal force acting on a blade during the mixing of a particulate material, they searched for the influence of dimensionless parameters on the mixing process described in (1). The results of the usage of DEM simulations were fundamental studies on the understanding of the behaviour of round particles during the mixing with a flat blade. The effect of the slope of the head of the blade as well as the blade speed of mixing was studied. They discovered that the slope of the head of the blade has a real influence on the shape and the height of the pile of the material in front of the mixing blade. With a slanted blade head, a high heap in the front of the blade was formed, where a static zone was also created. In the investigation of the turning of the blade head to $135^{\circ}$, they came to the conclusion that such a rotation significantly reduces the duration and height of the pile in front of the blade and that it is more suitable for mixing round particles. Horizontal forces acting on the blade were strongly affected by the blade head angle and were lessened by the increase of this slope. Due to the simulation, the authors showed that, with a constant blade head slope and with a constant depth of immersion, the horizontal force is not dependent on the blade speed during mixing. In the conclusion of their work, they tried to define the speed fields during the mixing and to apply them to the convective style of mixing, which would significantly reduce the calculation time.

The authors S. Radl, D. Brandl, H. Haimburg, B. J. Glasser and J. G. Khinast added this to their research work [12, in which they attempted to compare the flow of the fluid of the bulk material on the basis of computer simulations and simple experiments of the movement of the investigated material. For the fluid flow, they used the dimensionless Froud number and applied this number for the flow of the particulate material, by which they divided the moving bulk material into several areas simply according to the 


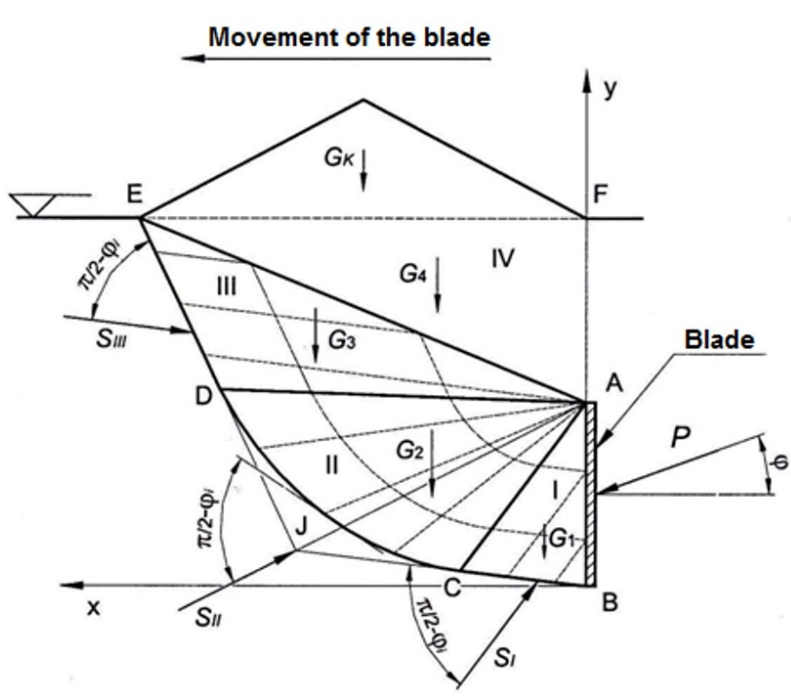

Figure 1. The Novosad model [13].

value of the Froud number. The authors came to the conclusion that the behaviour of the grained material can be described by the Froud number in analogy with the fluids. They found out that a recirculation zone is located in front of the blade and that this zone disappears when the critical value of the Froud number 0.5 is exceeded. The difference between the area beyond the blade in the mixing of particulate materials and fluids is caused by the rheology of granular materials. Furthermore, they came to the conclusion that if the mixing chamber is fuller, it leads to a significant change in the speed profile of the material before and after the moving blade, and that the recirculation zone disappears. The influence of the distance of the blade from the bottom of the vessel on the speed profiles was also significant, while a change in the slope angle of the blade did not have a significant effect on the speed of the particles in the upper part of the batch.

\section{ENERGY ASPECT OF THE MIXING PROCESS}

The energy aspect of the mixing process can be derived from the theory of a stress balance of a particle material and the theory of Mohr's circle. The Novosad model (Figure 1), which theoretically allows an approximate calculation of the force acting on the blade during mixing, also comes out of these theories. This model takes into consideration the effect of the mechanical-physical properties of the particulate material.

The calculation of the horizontal element of the force comes out of relation (3) for the calculation of the normal element of the burdening acting on the front side of the blade during a low blade immersion $(z / B \leq 1)$, where $z$ is the distance from the surface of the material to the lower edge of the blade and $B$ is the height of the blade) with consideration of the effect of the weight of the particulate material:

$$
\begin{aligned}
p_{m n}= & F_{M} q_{m} e^{2 \omega \tan \varphi_{i}}+\sigma_{C}\left(F_{M} e^{2 \omega \tan \varphi_{i}}-1\right) \\
& +\sigma_{C S}+ \\
& \frac{2}{3} \rho_{S} g B\left(J_{M} e^{3 \omega \tan \varphi_{i}}-S_{M}\right. \\
& \left.+T_{M}\left(M_{M} e^{3 \omega \tan \varphi_{i}}-N_{M}\right)\right),
\end{aligned}
$$

where

$$
\begin{aligned}
F_{M}= & \frac{\sin \mu_{A 2} \cos \mu_{P 2} \cos \varphi_{p 1}}{\cos \left(\mu_{A 1}+\varphi_{p 1}\right) \sin \mu_{P 1}}, \\
J_{M}= & \frac{\sin ^{2} \mu_{A 2} \sin \mu_{P 2} \cos \mu_{P 2} \cos \varphi_{p 1}}{\sin \left(\mu_{A 1}+\mu_{A 2}\right) \sin \mu_{P 1} \cos \left(\mu_{A 1}+\varphi_{p 1}\right)}, \\
S_{M}= & \frac{\sin ^{2} \mu_{A 1} \sin \mu_{A 2} \cos \varphi_{p 1}}{\sin \left(\mu_{A 1}+\mu_{A 2}\right) \cos \left(\mu_{A 1}+\varphi_{p 1}\right)}, \\
T_{M}= & \frac{1}{9 \tan ^{2} \varphi_{p 1}+1} \\
& \times \frac{\sin ^{2} \mu_{A 2} \cos \varphi_{p 1}}{\sin \left(\mu_{A 1}+\mu_{A 2}\right) \cos \left(\mu_{A 1}+\varphi_{p 1}\right) \cos \varphi_{i}}, \\
M_{M}= & 3 \sin \left(\mu_{A 1}+\omega\right) \tan \varphi_{i}-\cos \left(\mu_{A 1}+\omega\right), \\
N_{M}= & 3 \sin \mu_{A 1} \tan \varphi_{i}-\cos \mu_{A 1}
\end{aligned}
$$

The calculation of the normal el ment of the burdening acting on the front side of the blade during a medium blade immersion $(z / B>1)$ with consideration of the effect of the weight of the particulate material comes out of the following relation:

$$
\begin{aligned}
p_{m n}= & F_{S} q_{1 m} e^{2 \omega \tan \varphi_{i}}-q_{p}+ \\
& +\sigma_{C S} \frac{2}{3} \rho_{S} g B\left(R_{S} e^{3 \omega \tan \varphi_{i}}\right. \\
& \left.\quad-S_{S}+T_{S}\left(M_{S} e^{3 \omega \tan \varphi_{i}}-N_{S}\right)\right),
\end{aligned}
$$

where

$$
\begin{aligned}
F_{S}= & \frac{\sin \mu_{A 2} \cos \left(\mu_{P 2}-\varphi_{p 2}\right) \cos \varphi_{p 1}}{\cos \left(\mu_{A 1}+\varphi_{p 1}\right) \sin \mu_{P 1}}, \\
R_{S}= & \frac{\sin ^{2} \mu_{A 2} \sin \mu_{P 2} \cos \left(\mu_{P 2}-\alpha\right) \cos \varphi_{p 1}}{\sin \left(\mu_{A 1}+\mu_{A 2}\right) \sin \mu_{P 1} \cos \left(\mu_{A 1}+\varphi_{p 1}\right)}, \\
S_{S}= & \frac{\sin ^{2} \mu_{A 1} \sin \mu_{A 2} \cos \varphi_{p 1}}{\sin \left(\mu_{A 1}+\mu_{A 2}\right) \cos \left(\mu_{A 1}+\varphi_{p 1}\right)}, \\
T_{S}= & \frac{1}{9 \tan ^{2} \varphi_{p 1}+1} \\
& \times \frac{\sin ^{2} \mu_{A 2} \cos \varphi_{p 1}}{\sin \left(\mu_{A 1} \mu_{A 2}\right) \cos \left(\mu_{A 1}+\varphi_{p 1}\right) \cos \varphi_{i}}, \\
M_{S}= & 3 \sin \left(\mu_{A 1}+\omega\right) \tan \varphi_{i}-\cos \left(\mu_{A 1}+\omega\right), \\
N_{S}= & 3 \sin \mu_{A 1} \tan \varphi_{i}-\cos \mu_{A 1}
\end{aligned}
$$

For non-cohesive particulate materials, relations (3) and (10) also apply, substituting zero cohesion $\sigma_{C}=$ $0 \mathrm{~Pa}$ and $\sigma_{C S}=0 \mathrm{~Pa}$, in order to the pressure in the particulate material to safely rise over the value of the limit load, it is necessary to include in the calculation the safety factor $k$ in the calculation. In the case of a non-cohesive particulate material, $k=1.5$. The 


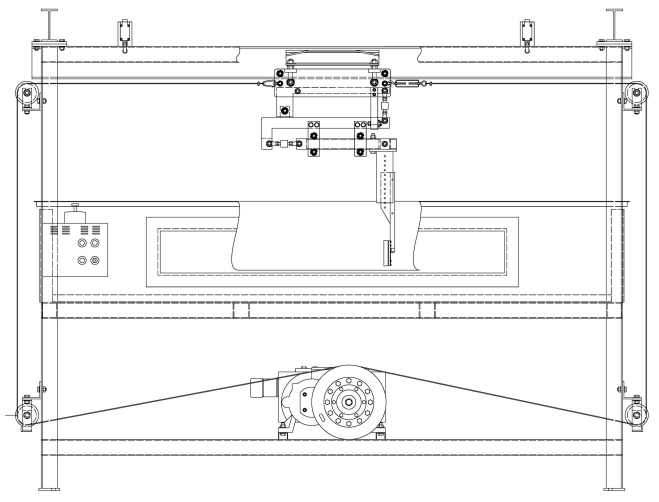

FIGURE 2. Scheme of experimental station.

\begin{tabular}{lc}
\hline Particulate material & $\begin{array}{c}\text { Granulated } \\
\text { polyethylene }\end{array}$ \\
\hline Diameter of particle* & $3.4 \mathrm{~mm}$ \\
Mass of particle & $2.13 \cdot 10^{-2} \mathrm{~g}$ \\
Volume of particle & $2.03 \cdot 10^{-8} \mathrm{~m}^{-3}$ \\
Density of partilce & $932.0 \mathrm{~kg} \mathrm{~m}^{-3}$ \\
Bulk density (tapped) & $607.9 \mathrm{~kg} \mathrm{~m}^{-3}$ \\
Angle of repose & $30.9^{\circ}$ \\
Angle of internal friction & $31.8^{\circ}$ \\
Angle of wall friction (steel) & $19.4^{\circ}$ \\
\hline
\end{tabular}

TABLE 1. Mechanical and physical properties of granulated polyethylene [16] (*equivalent diameter of particle by volume)

horizontal force component is calculated according to relation

$$
H_{T E O R}=k p_{m n}(B L) .
$$

The values of the horizontal force components acting on the blade found in this way are the average force values acting on the whole blade area.

\section{EXPERIMENTAL PART}

Experimental measures were performed on an experimental measuring station made up of a static chamber, a frame, the drive, the measuring element and a mixing blade. The mixing chamber includes a glass side wall for a visual control of events during the mixing. The mobile measuring element is equipped with two force sensors to record the total horizontal and vertical components of the force.

Granulated polyethylene was used for the experiment. The properties of granulated polyethylene are shown in Table 1. All the properties were measured by standard procedures [14, 15].

A set of measurements was performed for several blade immersion depths and different motion speeds of homogenizer blade motion.

\section{Simulation of DEM method PROCESS}

The DEM is a numerical method for the calculation of the pressure and shifting of the individual elements

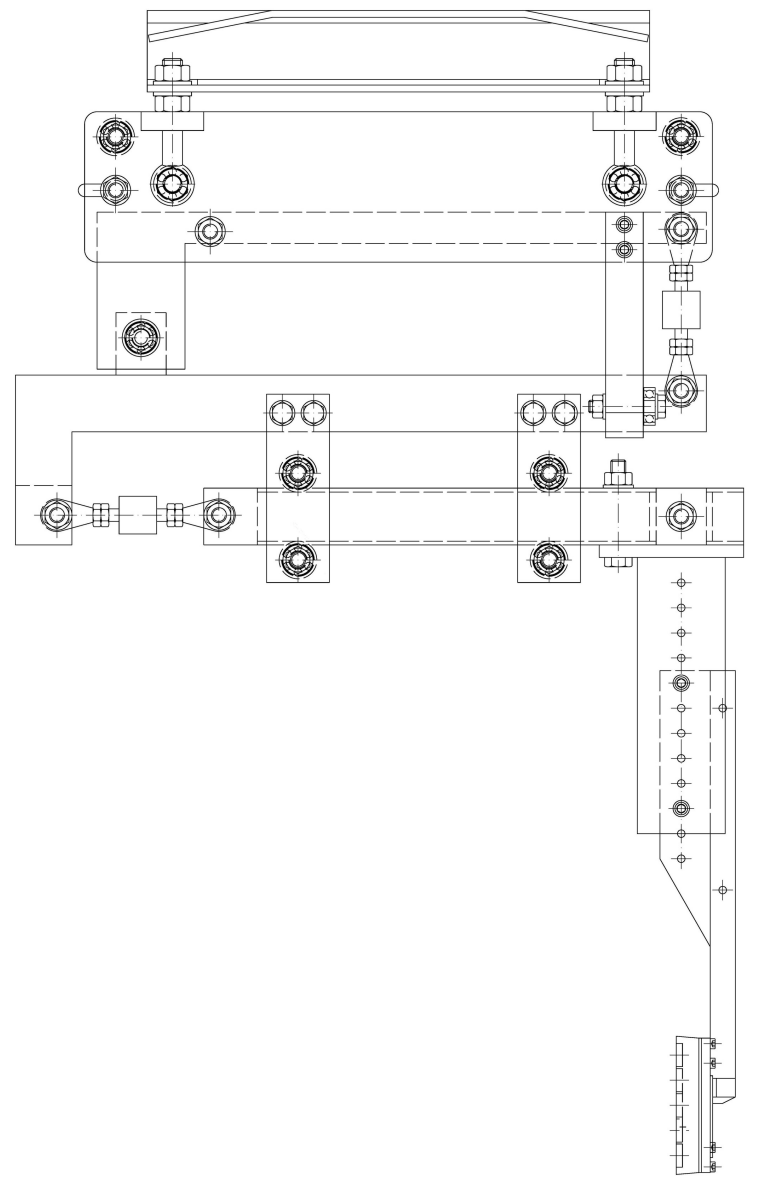

Figure 3. Detail of measuring element.

in a volume that contains a large amount of particles. A granular material is modelled as a set of solid particles and the interactions between the individual particles. The program used for the modelling of this task was EDEM Academic ${ }^{\mathrm{TM}}$ from the DEM Solution UK Company. The goal of the experimental simulation was to look at the fields of particles in front of a moving mixing element and to establish the average force acting on the blade due to the movement of the particulate material layer. The calculation method was Herz-Mindlin with RVD Rolling Friction. Round particles with the properties of the experimental material were used for the simulations. For simplification of the simulation, the width of the chamber was reduced, but it was considered as if the interaction between the particles had actually occurred in contact with the walls. The properties of the materials and their interactions are shown in Tables 1 and 2

The simulations were performed for 4 blade immersions at a speed of $0.2 \mathrm{~m} \mathrm{~s}^{-1}$. In Figures 4, 5, 6 and 7 a cross-section of the mixing chamber for the investigated blade immersion depths with marking of the individual layers of the particulate material can be seen [17].

Graphic behaviours of the particle movement before and after the mixing element for the investigation of the blade immersion depth are shown in Figures 8, 9 , 10 and 11. 


\begin{tabular}{lcccc}
\hline \multicolumn{1}{c}{ Model material } & Poisson's ratio & Solids density & Shear modulus \\
\hline Steel (mixing chamber) & 0.3 & $7800 \mathrm{~kg} \mathrm{~m}^{-3}$ & $8 \cdot 10^{10} \mathrm{~Pa}$ \\
Steel (mixing blade) & 0.3 & $7800 \mathrm{~kg} \mathrm{~m}^{-3}$ & $8 \cdot 10^{10} \mathrm{~Pa}$ \\
Granulated polyethylene & 0.245 & $932 \mathrm{~kg} \mathrm{~m}^{-3}$ & $1.3 \cdot 10^{8} \mathrm{~Pa}$ \\
\hline \multicolumn{2}{c}{ Interaction } & \multicolumn{3}{c}{ Coefficient } \\
\cline { 2 - 5 } & restitution & static & rolling \\
& & friction & friction \\
\hline & & 0.5 & 0.62 & 0.1 \\
Granulated polyethylene - Granulated polyethylene & 0.35 & 0.35 & 0.1 \\
Granulated polyethylene - Steel (mixing blade) & Granulated polyethylene - Steel (mixing chamber) & 0.35 & 0.62 & 0.1 \\
\hline
\end{tabular}

TABLE 2. Properties of the materials and their interactions entered in the program EDEM Academic ${ }^{\mathrm{TM}}$

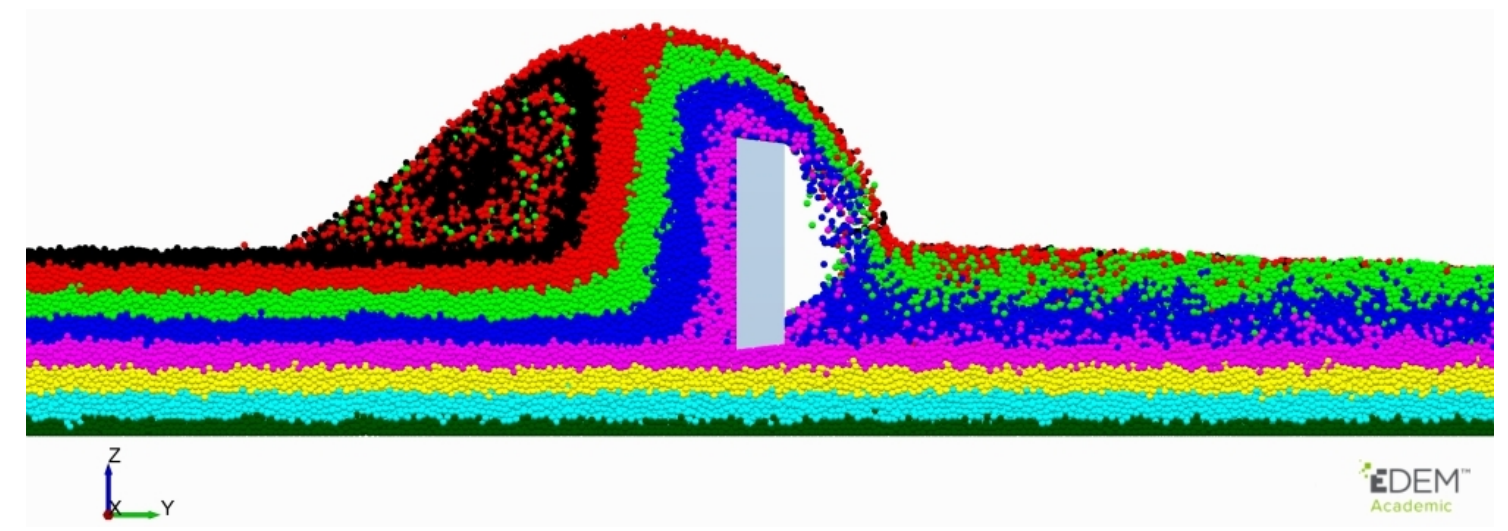

Figure 4. Cross-section of mixing chamber with dimensionless immersion of blade $z / B=0.5$ at blade speed $0.2 \mathrm{~ms}^{-1}$ in program EDEM Academic ${ }^{\mathrm{TM}}$.

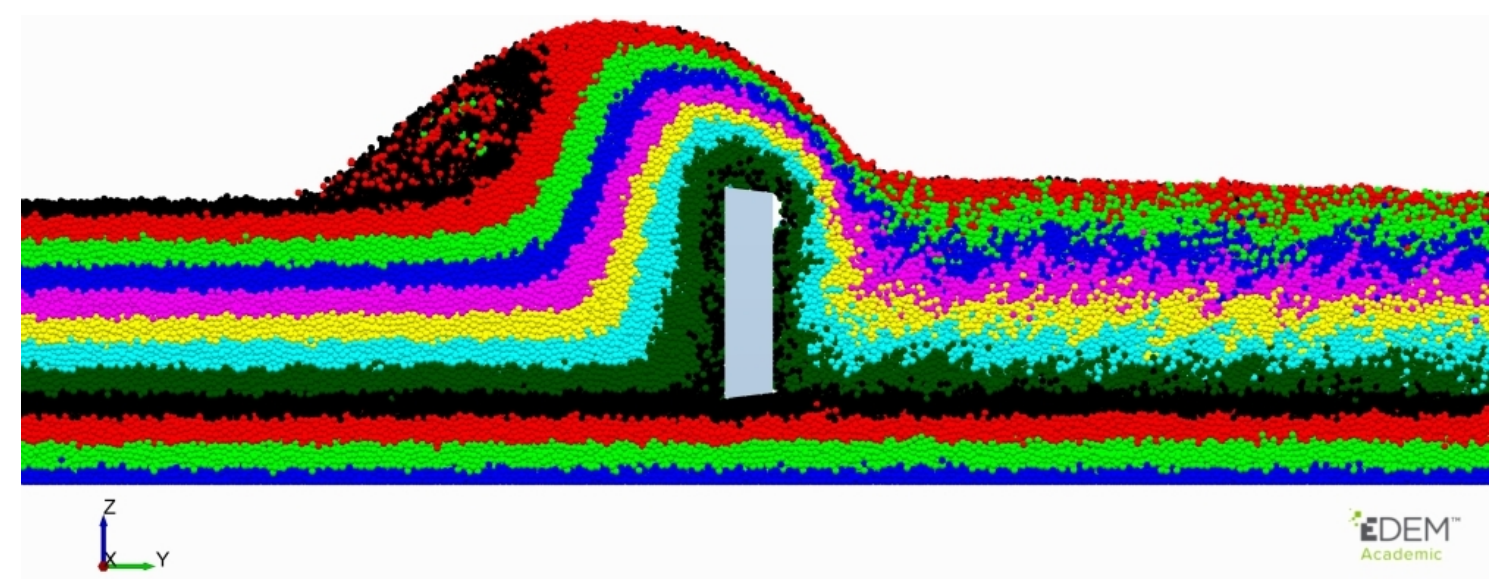

Figure 5. Cross-section of mixing chamber with dimensionless immersion of blade $z / B=1.0$ at blade speed $0.2 \mathrm{~ms}^{-1}$ in program EDEM Academic ${ }^{\mathrm{TM}}$. 


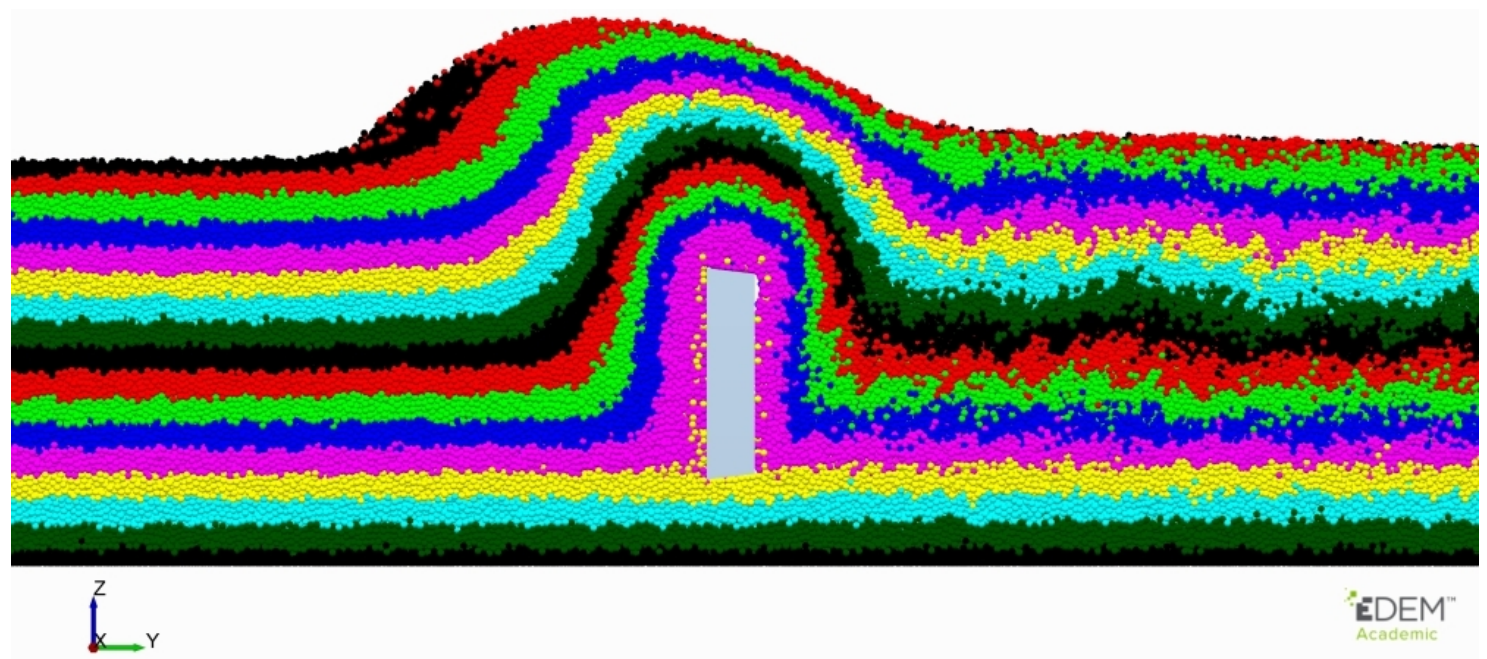

FiguRE 6 . Cross-section of mixing chamber with dimensionless immersion of blade $z / B=1.5$ at blade speed $0.2 \mathrm{~ms}^{-1}$ in program EDEM Academic ${ }^{\mathrm{TM}}$.

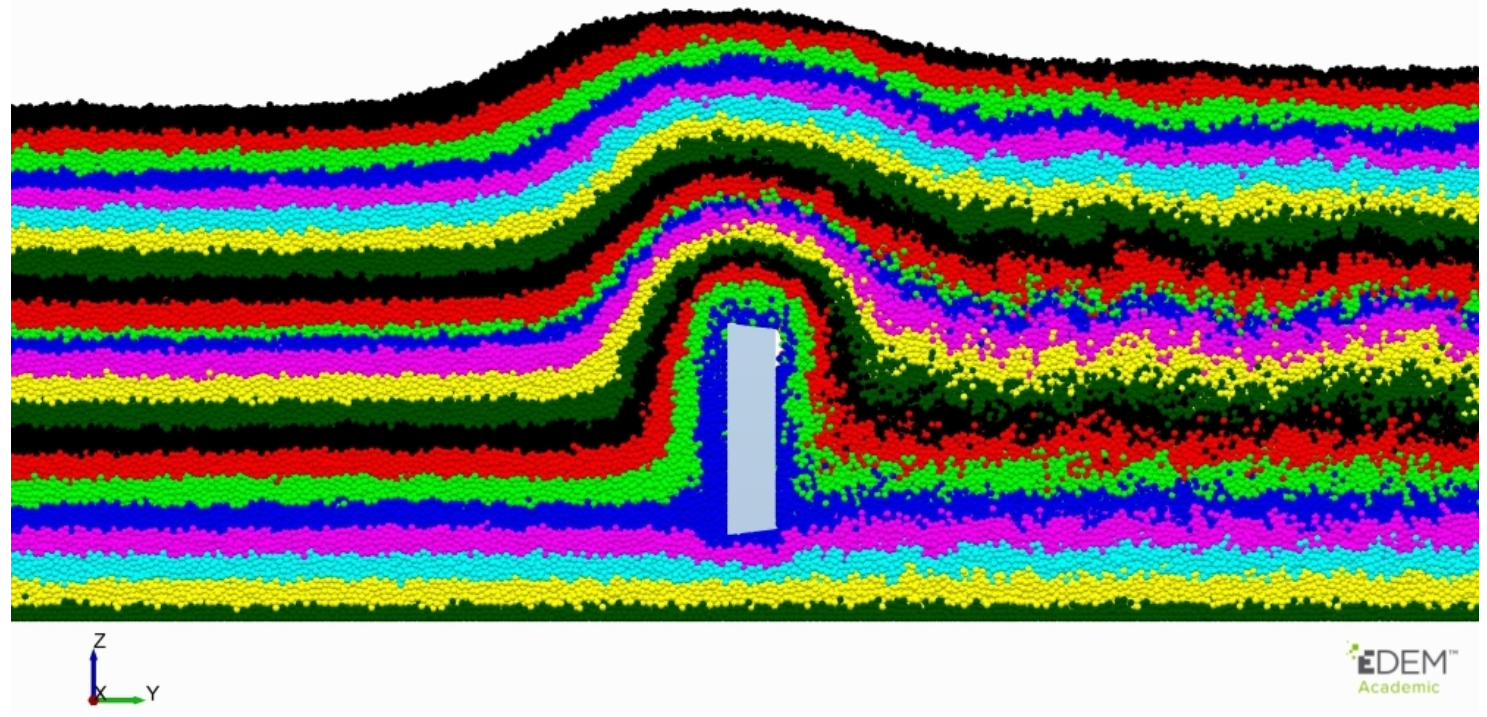

Figure 7. Cross-section of mixing chamber with dimensionless immersion of blade $z / B=2.0$ at blade speed $0.2 \mathrm{~m} \mathrm{~s}^{-1}$ in program EDEM Academic ${ }^{\mathrm{TM}}$.

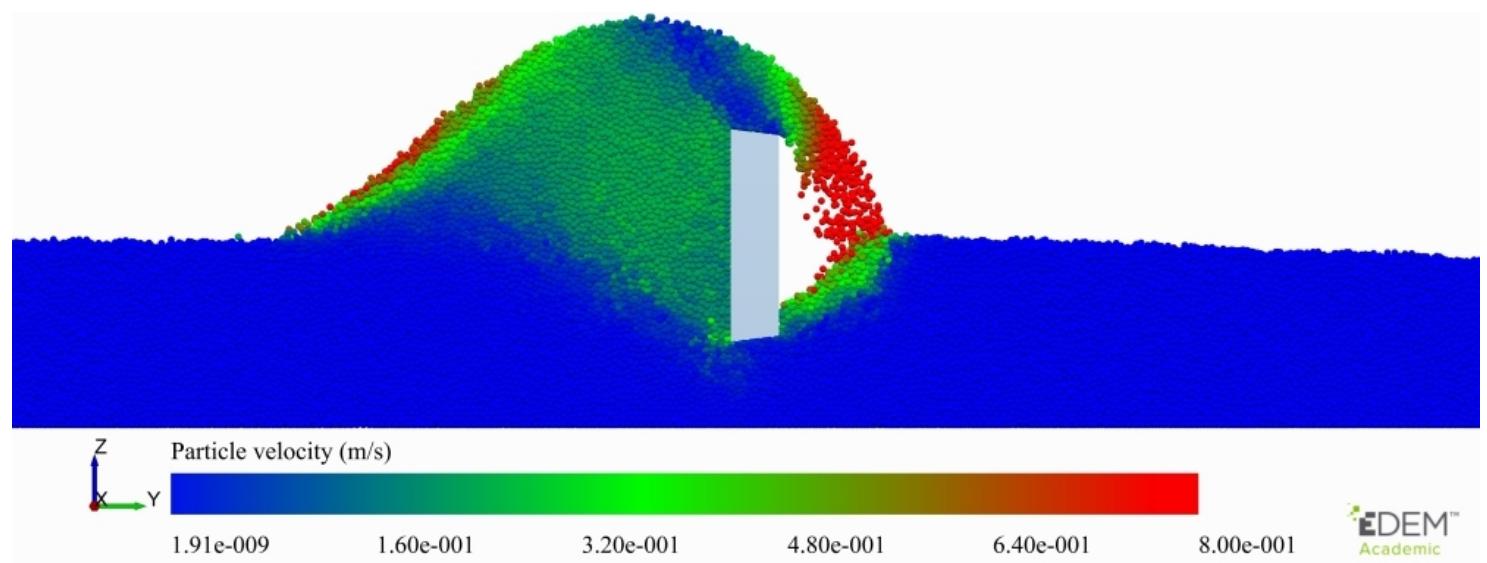

FiguRE 8. Graphic illustration of the movement of particles during mixing in mixing cross-section with dimensionless immersion of blade $z / B=0.5$ at blade speeds $0.2 \mathrm{~m} \mathrm{~s}^{-1}$ in the program EDEM Academic ${ }^{\mathrm{TM}}$. 


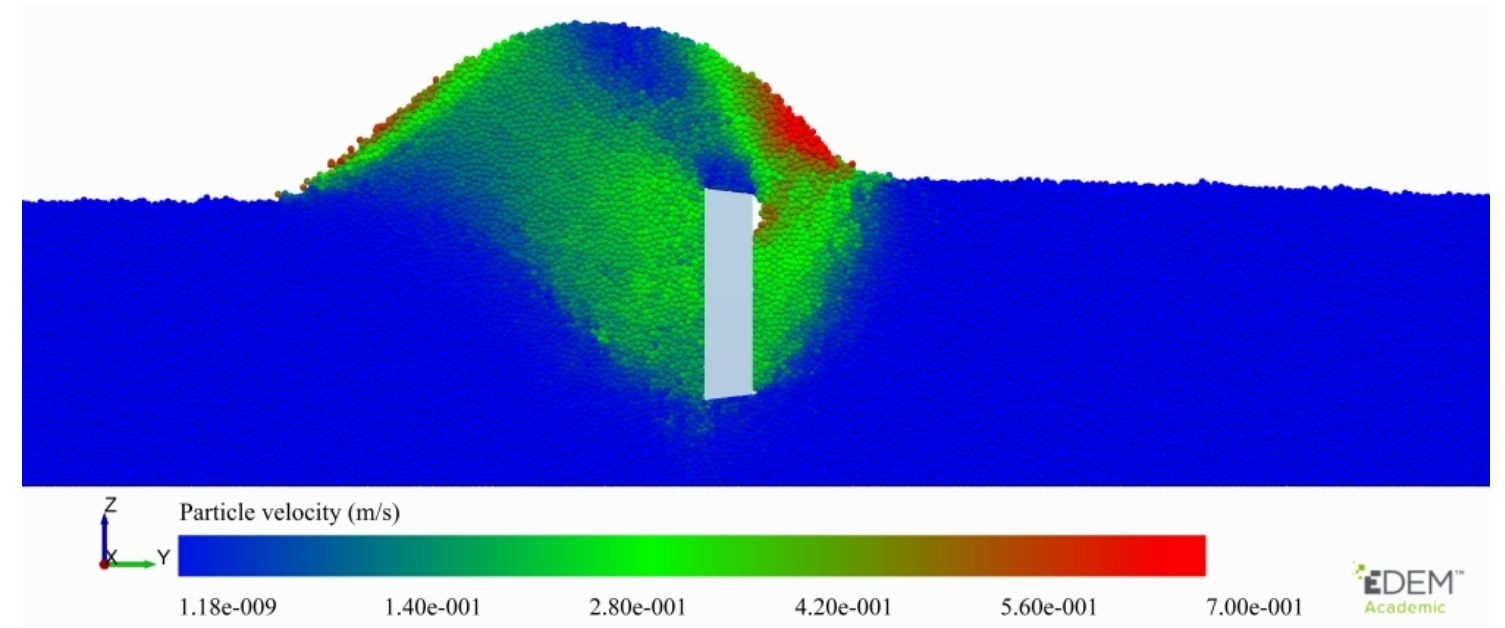

Figure 9. Graphic illustration of the movement of particles during mixing in mixing cross-section with dimensionless immersion of blade $z / B=1.0$ at blade speeds $0.2 \mathrm{~m} \mathrm{~s}^{-1}$ in the program EDEM Academic ${ }^{\mathrm{TM}}$.

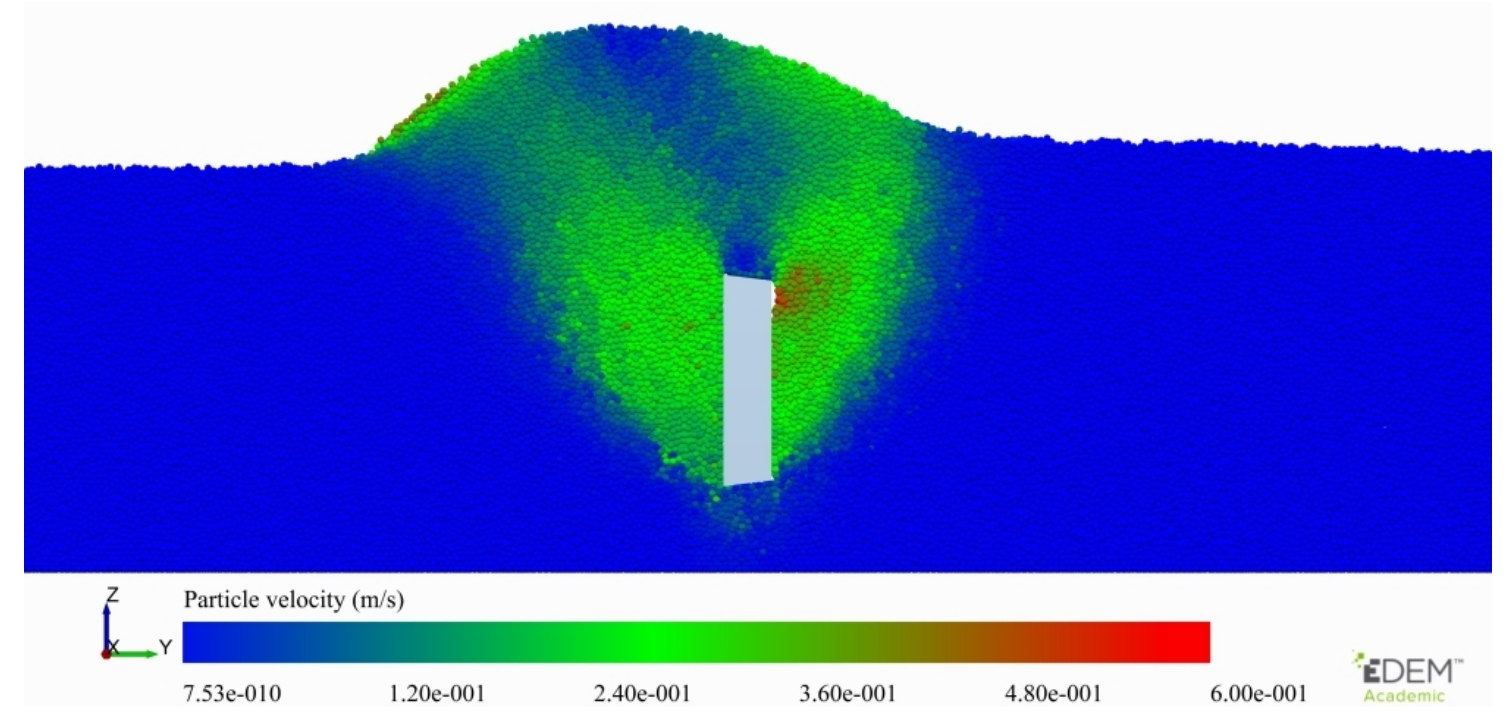

Figure 10. Graphic illustration of the movement of particles during mixing in mixing cross-section with dimensionless immersion of blade $z / B=1.5$ at blade speeds $0.2 \mathrm{~m} \mathrm{~s}^{-1}$ in the program EDEM Academic ${ }^{\mathrm{TM}}$.

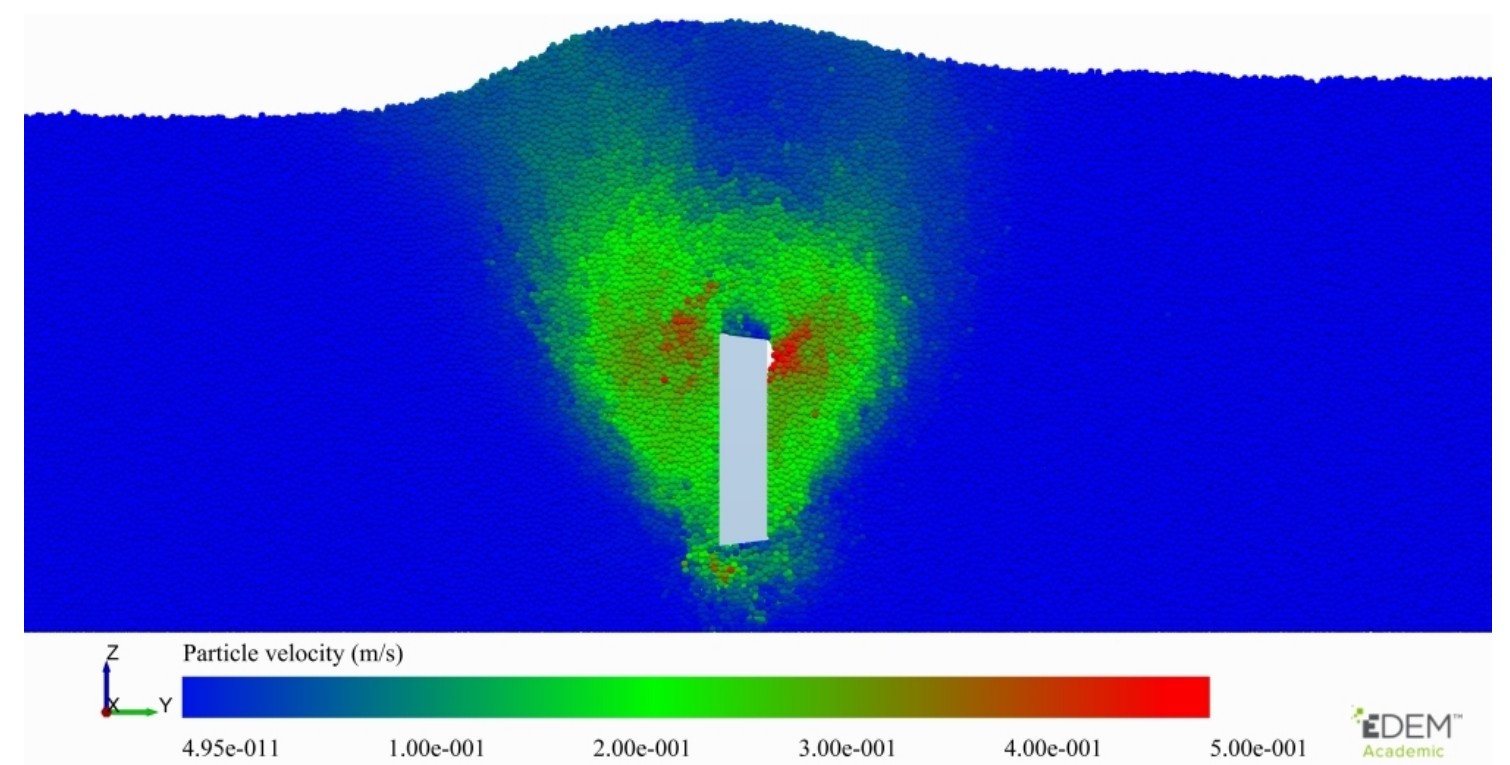

FIGURE 11. Graphic illustration of the movement of particles during mixing in mixing cross-section with dimensionless immersion of blade $z / B=2.0$ at blade speeds $0.2 \mathrm{~m} \mathrm{~s}^{-1}$ in the program EDEM Academic ${ }^{\mathrm{TM}}$. 


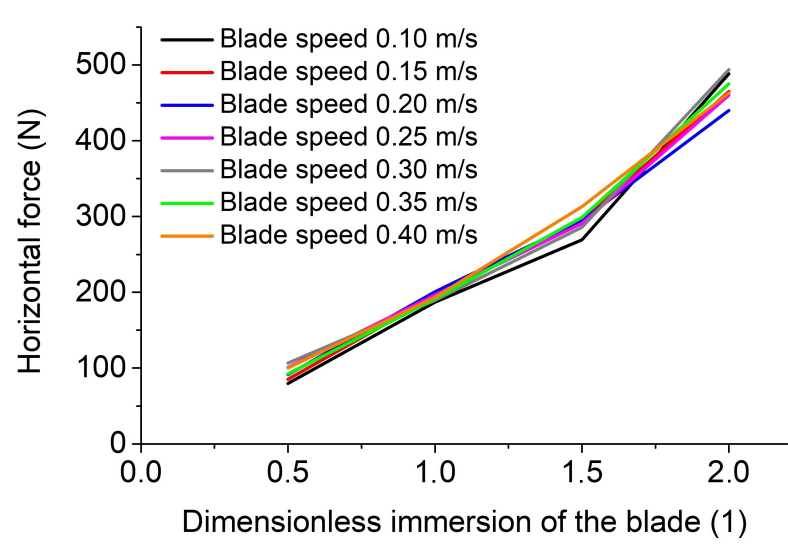

Figure 12. Dependency of horizontal force on dimensionless blade immersion.

\section{Results}

\subsection{INFLUENCE OF BLADE SPEED ON HORIZONTAL FORCE}

From the measured data, it follows that the force by which the particulate material acts on the moving blade at a given speed depends only slightly on its value for all blade immersions. This assumption was confirmed experimentally by Š. Gužela in the work 18 and K. Kravárik in the work 8] where they stated that this influence is also more expressive for speeds higher than the speed of the blade. The dependency of the horizontal force on the blade immersion for the investigated speeds is shown in Figure 12 .

\subsection{INFLUENCE ON DEPTH OF BLADE IMMERSION ON HORIZONTAL FORCE}

From the measured data, it follows that the force by which the particulate material acts on the moving blade at a given speed depends, to a significant degree, on the immersion of the blade in the batch of the particulate material. A graphic illustration of the dependency of the horizontal force acting on the blade during the mixing on the speed for the given immersion in a batch is shown in Figure 13. From the experiments, it is clear that the depth of the blade immersion is a significant parameter affecting the size of the horizontal force acting on the blade. With the increasing depth, this force component increases.

\subsection{COMPARISON OF RESUlting HORIZONTAL FORCE ACTING ON A BLADE}

The comparison of the resulting horizontal force acting on a blade for individual blade immersion depths during mixing at blade speed $0.2 \mathrm{~m} \mathrm{~s}^{-1}$ is shown in Figure 14. The calculated value of the horizontal force according to the Novosad model was experimentally measured on a HBM U9B sensor. The reworked measured values were also compared on ceramic membrane pressure sensors, with this procedure carried out according to [19] and stated in [13].

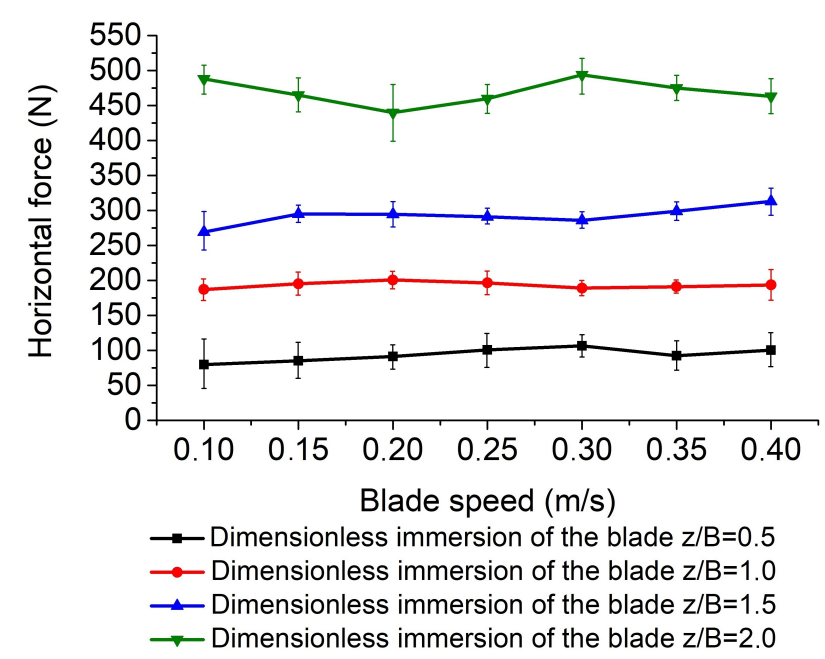

FiguRE 13. Dependency of horizontal force on blade speed during mixing in investigated dimensionless blade immersions in batches of particulate material.

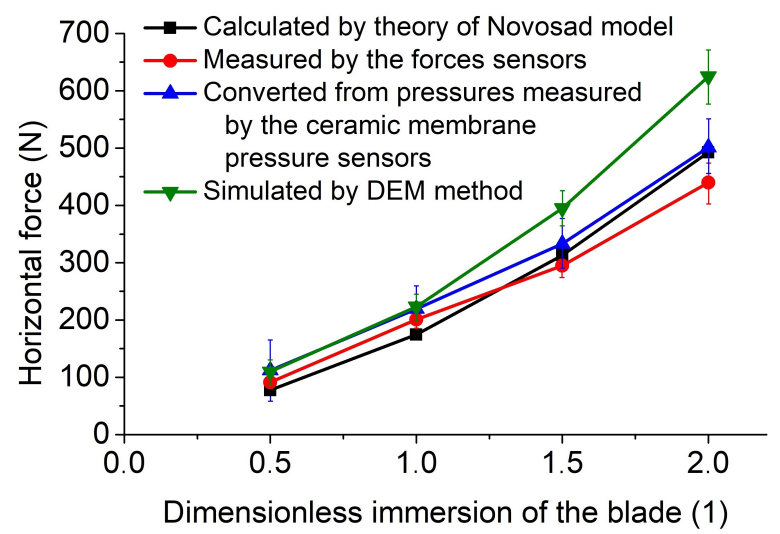

Figure 14. Dependency of horizontal force on blade speed during mixing in investigated dimensionless blade immersions in batches of particulate material.

\section{Conclunsion}

The experiments performed on the mixing of particulate materials confirmed that the behaviour of a material during the mixing is in conformity with the theoretical knowledge and simulations by the DEM method in the EDEM Academic ${ }^{\mathrm{TM}}$ program. The horizontal force acting on a homogenizer blade was used for the comparison of theoretical calculations, experimental measurements and simulation. The measured values of the horizontal force were in excellent conformity with the theory and these experiments can be considered successful, as Figure 14 shows.

The DEM simulations performed for the support of the theoretical knowledge and experimental measures demonstrated the contemporary trend of using calculation models, while the DEM method for monitoring the movement of individual particles showed to be suitable. Through the use of this method, it was possible to observe the movement and behaviour of the particles in front of and behind the mixing element over time, as it is presented in Figures 8,9 10 and 11 . For confirming the accuracy of the move- 

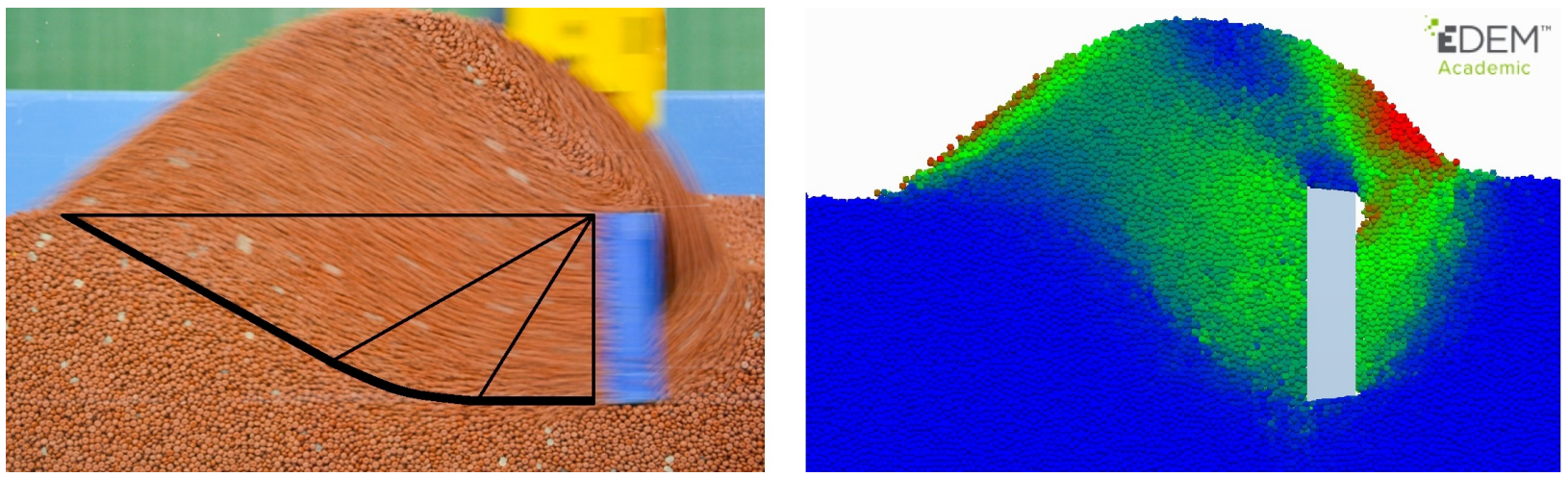

FIGURE 15. Photographic illustration of particle movement during mixing in a mixing chamber with dimensionless blade immersion $z / B=1.0$ at blade speed $0.2 \mathrm{~m} \mathrm{~s}^{-1}$ in program EDEM Academic ${ }^{\mathrm{TM}}$ (legend: areas delineated by red lines show the individual slip areas in front of a moving blade constructed according to the Novosad model, and the thick black line illustrates the limit slip line constructed according to the Novosad model).

ment of the individual particles in simulations, images were also created by photographic methods that compared the movement of the particles in front of and behind the mixing element. A comparison of particle movement in the DEM method and in experiments at $z / B=1.0$ and blade movement speed $0.2 \mathrm{~m} \mathrm{~s}^{-1}$ is shown in Figure 15 .

\section{LIST OF SYMBOLS}

$\mu_{A 1}$ Deflection angle of slide lines in the active state of limit equilibrium from the load plane $\left[^{\circ}\right]$

$\mu_{A_{2}}$ Deflection angle of slide lines in the active state of limit equilibrium from the load plane $\left[^{\circ}\right]$

$\mu_{P_{1}}$ Deflection angle of slide lines in the passive state of limit equilibrium from the load plane $\left[^{\circ}\right]$

$\mu_{P_{2}}$ Deflection angle of slide lines in the passive state of limit equilibrium from the load plane $\left[^{\circ}\right]$

$\rho_{S} \quad$ Bulk density $\left[\mathrm{kg} \mathrm{m}^{-3}\right]$

$\sigma_{C} \quad$ Normal cohesion of experimental material $[\mathrm{Pa}]$

$\sigma_{C S}$ Normal cohesion contact between experimental material and construction material $[\mathrm{Pa}]$

$\varphi$ Angle of wall friction between experimental particulate material and steel $\left[^{\circ}\right]$

$\varphi_{i} \quad$ Angle of internal friction $\left[^{\circ}\right]$

$\varphi_{p 1} \quad$ Virtual angle of wall friction particulate material contained in the active state of limit equilibrium [ $\left.{ }^{\circ}\right]$

$\omega$ Rotation angle of slide lines in the transition area $\left[^{\circ}\right]$

$g$ Acceleration due to gravity $\left[\mathrm{m} \mathrm{s}^{-2}\right]$

$k$ Safety factor [1]

$p_{m n}$ Normal component of the limit uniform load $[\mathrm{Pa}]$

$r_{w}$ Radius of logarithm spiral [m]

$q_{p} \quad$ Uniform surface load $[\mathrm{Pa}]$

$q_{1 m}$ Virtual surrounding load acting on the passive areain particulate material $[\mathrm{Pa}]$

$z \quad$ Immersion of the blade [m]

$B$ Blade height $[\mathrm{m}]$

$F_{M}$ Dimensionless coefficient for the shallow immersion of the blade [1]

$F_{S}$ Dimensionless coefficient for the medium immersion of the blade [1]
$H_{T E O R}$ Horizontal force acting on the blade calculated by theory $[\mathrm{N}]$

$J_{M}$ Dimensionless coefficient for the shallow immersion of the blade [1]

$L$ Blade length $[\mathrm{m}]$

$M_{M}$ Dimensionless coefficient for the shallow immersion of the blade [1]

$M_{S}$ Dimensionless coefficient for the medium immersion of the blade [1]

$N_{M}$ Dimensionless coefficient for the shallow immersion of the blade [1]

$N_{S}$ Dimensionless coefficient for the medium immersion of the blade [1]

$R_{S}$ Dimensionless coefficient for the medium immersion of the blade [1]

$S_{M}$ Dimensionless coefficient for the shallow immersion of the blade [1]

$S_{S}$ Dimensionless coefficient for the medium immersion of the blade [1]

$T_{M}$ Dimensionless coefficient for the shallow immersion of the blade [1]

$T_{S}$ Dimensionless coefficient for the medium immersion of the blade [1]

\section{ACKNOWLEDGEMEnTS}

This article was created with the support of the Ministry of Education, Science, Research and Sport of the Slovak Republic within the Research and Development Operational Programme for the project "University Science Park of STU Bratislava", ITMS 26240220084, co-funded by the European Regional Development Fund.

The authors wish to acknowledge the Scientific Grant Agency of the Ministry of Education, Science, Research and Sport of the Slovak Republic and the Slovak Academy of Sciences for the financial support of this research by grant VEGA 1/0276/17.

\section{REFERENCES}

[1] J. Nečas, J. Hlosta, D. Žurovec, et al. Feeder type optimisation for the plain flow discharge process of an underground hopper by discrete element modelling. Advances in Science and Technology 11(3):246-252, 2017. DOI:10.12913/22998624/76100 
[2] J. Rozbroj, J. Nečas, D. Žurovec, et al. Simulation of material flow through a sample divider. Advances in Science and Technology 12(1):194-199, 2018. DOI:10.12913/22998624/85663

[3] D. F. Bagster. The prediction of the force needed to move blades through a bed of cohesionless granules. Powder Technology 3:153-162, 1969/70. DOI:10.1016/0032-5910(69)80067-7

[4] D. F. Bagster, J. Bridgwater. The flow of granular material over a moving blade. Powder Technology 3:323-338, 1969/70. DOI:10.1016/0032-5910(69)80104-X

[5] D. F. Bagster, J. Bridgwater. The measurement of the force needed to move blades through a bed of cohesionless granules. Powder Technology 1:189-198, 1969. DOI:10.1016/0032-5910(67)80036-6

[6] J. Novosad. Mechanika sypkých hmot. Praha: Institut pro výchovu vedoucích pracovniků ministerstva průmyslu, 1983. (In Czech).

[7] J. Novosad. Studies on Granular Materials. I. Kinematics of granular materials mixed by mechanical impeller. Praha: Československá akadémia vied, 1963.

[8] K. Kravárik. Energetické aspekty miešania partikulárnych látok. Dissertations thesis. STU, Faculty of Mechanical Engineering, Bratislava, 1999. (In Slovak).

[9] Y. Muguruma, T. Tanaka, S. Kawatake, Y. Tsuji. Discrete particle simulation of a rotary vessel mixer with baffles. Powder Technology 93:261-266, 1997. DOI:10.1016/S0032-5910(97)03281-6

[10] M. S. Siraj, S. Radl, B. J. Glasser, J. G. Khinast. Effect of blade angle and particle size on powder mixing performance in a rectangular box. Powder Technology 211:100-113, 2011. DOI:10.1016/j.powtec.2011.04.004
[11] G. R. Chandratilleke, A. B. Yu, J. Bridgwater. A dem study of the mixing of particles induced by a flat blade. Chemical Engineering Science 79:54-74, 2012. DOI:10.1016/j.ces.2012.05.010

[12] S. Radl, D. Brandl, H. Heimburg, et al. Flow and mixing of granular material over a single blade. Powder Technology 226:199-212, 2012. DOI:10.1016/j.powtec.2012.04.042

[13] P. Peciar, M. Peciar, R. Fekete. Analysis of mixing of particulate material by a single blade. Solid State Phenomena 244:40-47, 2016. ISSN 1012-0394.

[14] D. Geldart, E. Abdullah, A. Verlinden. Characterisation of dry powders. Powder Technology 190:70-74, 2009. DOI:10.1016/j.powtec.2008.04.089

[15] D. McGlinchey. Characterisation of Bulk Solids. Wiley-Blackwell, 2009. ISBN: 978-1-405-14363-9.

[16] P. Peciar. Pressure Distribution on the Particulate Material Homogenizer Blade. Dissertations thesis. STU, Faculty of Mechanical Engineering, Bratislava, 2014. ISBN 978-80-227-4146-0 (In Slovak).

[17] P. Peciar. Research of the selected processes of particulate materials processing. Habilitation thesis. STU, Faculty of Mechanical Engineering, Bratislava, 2018. ISBN 978-80-227-4801-8 (In Slovak).

[18] Š. Gužela, M. Peciar. Analýza napätia pôsobiaceho na lopatku postupujúcu vrstvu partikulárnej látky. STU, Faculty of Mechanical Engineering, Bratislava, 2006. ISBN: 80-227-2443-2, (In Slovak).

[19] P. Peciar, M. Peciar, R. Fekete. Mobile universal measuring system of pressure in particulate material and calibration method of pressure sensors. Utility model no. 7283, Application no. 5068-2014. Industrial Property Office of the Slovak Republic, Banská Bystrica, Slovakia, 2015. 